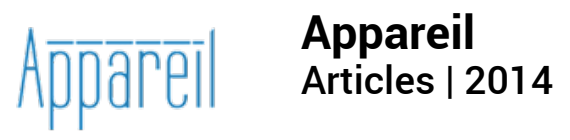

\title{
Violence fondatrice, mémoires de la dictature et politiques de la reconnaissance
}

\author{
Ricardo Salas Astrain
}

\section{(2) OpenEdition}

\section{Journals}

\section{Édition électronique}

URL : http://journals.openedition.org/appareil/1977

DOI : 10.4000/appareil.1977

ISSN : 2101-0714

\section{Éditeur}

MSH Paris Nord

\section{Référence électronique}

Ricardo Salas Astrain, « Violence fondatrice, mémoires de la dictature et politiques de la reconnaissance », Appareil [En ligne], Articles, mis en ligne le 06 mars 2014, consulté le 30 juillet 2020. URL : http://journals.openedition.org/appareil/1977 ; DOI : https://doi.org/10.4000/appareil.1977

Ce document a été généré automatiquement le 30 juillet 2020.

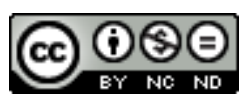

Appareil est mis à disposition selon les termes de la Licence Creative Commons Attribution - Pas d'Utilisation Commerciale - Pas de Modification 4.0 International. 


\title{
Violence fondatrice, mémoires de la dictature et politiques de la reconnaissance
}

\author{
Ricardo Salas Astrain
}

\section{NOTE DE L'AUTEUR}

Ce travail s'inscrit dans le cadre d'un projet FONDECYT n 1120701, “Teorías

contemporáneas del reconocimiento”, dont je suis le responsable.

\section{Prologue}

1 Notre approche de la dictature chilienne à travers le prisme d'une violence fondatrice qui traverse la société chilienne et les sociétés latino-américaines ne cherche en aucune façon à justifier un certain déterminisme historique au nom duquel le « pouvoir factuel militaire ou armé » serait un attribut inévitable ou nécessaire de la définition du pouvoir; il s'agit plutôt de tenter une manœuvre philosophique capable de saisir les faits politiques à partir d'une logique de la violence, en recourant, dans le cas spécifique de ce travail, à trois « figures » principales du pouvoir tel qu'il est défini par les péripéties de la violence structurelle en terres américaines. Nous nous référons à la violence du conquérant, à la violence des indépendances propres aux États naissants et à leurs guerres postérieures ainsi qu'à la violence de l'État contre les civils, définie par la doctrine de la sécurité nationale.

Dans cette perspective, il s'agit d'assumer une longue histoire de guerres, d'horreurs et d'assassinats associés à la constitution des pouvoirs les plus divers, histoire dont les conséquences tragiques sont encore vécues de nos jours par les sujets et les communautés. Celle du Chili est une histoire qui dissimule les effets de la recomposition permanente des pouvoirs de fait, où le pouvoir militaire chilien a joué 
un rôle prépondérant, comme l'atteste l'image de la «Flandre indienne », ou bien celle d'une " armée victorieuse et jamais vaincue ». La militarisation de la société coloniale et $\mathrm{du}$ naissant pays républicain est un fait décisif, qui tient son rôle dans la configuration de la démocratie chilienne. Les Chiliens ne représentent pas une exception comme cela a pu être prétendu dans diverses historiographies; dans le cas du Chili, l'État fort est plutôt associé à la violence et au pouvoir des élites, tout en étant lié à des sociétés asymétriques configurées par la violence du pouvoir. En conséquent, selon une perception sociologique des modes de résolution des controverses, la violence n'est ni fortuite ni sporadique. Cela nous renvoie à l'emblématique et non moins controversée devise de la patrie chilienne : « Pour la raison ou pour la force ».

3 Ceci étant, il ne s'agit pas non plus de considérer la vie politique comme simple résultante de la violence et de succomber à une certaine "séduction de la violence " caractéristique d'un courant européen intellectuel de gauche. En parlant de « violence fondatrice ", nous souhaitons souligner - comme cela a déjà été signalé par la pensée politique actuelle - que le pouvoir politique héberge quelque chose d'ambigu et d'invisible, que l'on peut déceler derrière certains de ses traits hétérogènes et asymétriques à certains moments de l'histoire de nos nations ; c'est à ces moments que font irruption les pouvoirs factuels. Nous percevons en conséquent une permanente tension entre l'idéalité et la réalité, ce qui fait le propre d'une philosophie politique située.

4 Cette tension fera partie de l'analyse historique de notre trame politique. Cependant, nous proposons ici, comme proposition méthodologique, de ne pas nous concentrer sur de tels processus empiriques déjà soumis à des disciplines scientifiques - comme l'histoire, la science politique, la sociologie, etc.-, mais de développer une phénoménologie du pouvoir capable de rendre compte d'une structure de la sociabilité. En effet, il nous intéresse ici d'analyser comment la violence et le pouvoir alimentent en permanence l'ambiguïté entre les faits et les idéaux. Nous dévoilerons ici seulement quelques traits de ces trois figures de la violence, prises entre le politique et la politique en contexte latino-américain, en reprenant des figures déjà caractérisées dans d'autres travaux: l'invasion de l'Indo-Amérique et le traumatisme de la conquête; le couple civilisation-barbarie et la lutte de nationalité ; et la défense de l'État occidental avec la lutte anti-insurrectionnelle ${ }^{1}$.

\section{Penser la violence fondatrice et l'autonomie du politique}

5 Les principales idées que nous souhaitons développer ici partent d'une approche explicitement politique de la théorie de la reconnaissance, thème actuellement situé au cœur de la recherche philosophique, qui se préoccupe non seulement de définir les relations d'égalité et de différence mais interroge aussi comment s'établissent les formes d'humiliation et d'exclusion qui structurent les relations sociales. Selon la théorie physique de la reconnaissance, si l'on s'en réfère à la proposition d'A. Honneth, les relations de rencontre et de non-rencontre se situent dans des sphères différenciées de celles de l'amour, du droit et de la solidarité. La violence établie intervient quant à elle dans le quotidien, le juridique et l'économique. En ce sens, nous proposons d'étudier comment l'immense mouvement répressif mené depuis le coup d'état militaire s'en est pris, non seulement aux sujets qui luttaient pour une amélioration de 
la démocratie chilienne, comme les militants des partis et les dirigeants des mouvements sociaux, mais également à d'innocents civils et même à l'ensemble de la société chilienne.

La dictature militaire s'inscrit dans une profonde "pathologie de la raison politique ", concrétisée dans le mépris de la vie, l'humiliation des personnes et la violation systématique des droits de l'homme, avec le soutien silencieux d'une majorité qui profitait de cette discipline de la société. La négation de l'autre et l'oppression physique et psychologique devinrent une constante de la vie sociale chilienne au point de se convertir en une pratique répressive tolérée et acceptée par l'ensemble des élites économiques. Ces graves manifestations de violence ont généré une culture du silence, d'acceptation des méthodes d'exception et du discrédit d'un imaginaire de la lutte sociale, que très peu de secteurs de la société chilienne cherchent à remettre en cause.

7 Notre actuelle réflexion sur la violence politique prolonge, d'une certaine façon, une hypothèse antérieurement développée où nous analysions le phénomène des "animitas" à partir de la notion de violence dans le monde populaire. Nous y développions une idée de R. Girard sur la violence comme objet central, afin de comprendre comment les populations et paysans pauvres conçoivent le sacré2. En l'espèce, en révisant ces hypothèses, nous proposons d'avancer vers une formulation plus adaptée à la philosophie politique et d'explorer l'idée que la violence ne touche pas seulement le monde du sacré populaire mais concerne aussi les structures et les institutions du monde profane du pouvoir, c'est-à-dire le monde sociopolitique et socioculturel où se nouent les relations du quotidien.

8 Nous souhaiterions surtout démontrer, en suivant les pas de Paul Ricœur dans son célèbre article "Le Paradoxe de la politique ", que la nomenclature existante ne nous permet pas de comprendre les phénomènes politiques et qu'il est nécessaire de distinguer clairement le politique de la politique ; le premier se référant à la face idéelle du pouvoir et l'autre à son expression factuelle. Un érudit de la question nous signale à ce sujet :

En dépit de toute rationalité, il y a toujours au sein de l'État une violence résiduelle implantée, pour ainsi dire, par l'autorité à laquelle est garantie la capacité de décision. Et c'est à partir de ces caractéristiques violentes que peuvent surgir de nouveaux états de violence, qui s'inscrivent dans la structure même du politique. ${ }^{3}$

En d'autres termes, nous souhaitons démontrer que la violence systématique, qui apparaît dès le début du coup d'État militaire, il y a quarante ans, et qui conduit les institutions de l'État à mépriser systématiquement la vie humaine et les droits de ses citoyens, ne doit pas être perçue uniquement comme une simple orchestration venue de l'extérieur ou encore être considérée sous l'angle de la diabolisation des agents individuels ayant tenu des rôles majeurs dans cette guerre sale, mais doit aussi être envisagée comme un type de pouvoir déjà constitué et une violence structurelle déjà préalablement installée au sein de l'État. S'il existe un lien entre la violence ancestrale que nous étudions dans l'imaginaire du sacré populaire et la violence politique de la société chilienne post-coup d'État, c'est parce que la violence s'inscrit comme un phénomène constitué qui affecte l'ensemble des êtres humains dans leurs relations objectives et subjectives. Il n'est pas possible d'échapper à la violence; la violence qui anime l'État gouverné par les dirigeants militaires ne se circonscrit pas au domaine des forces militaires et à leurs appareils de répression, mais règne au sein de la société 
chilienne : c'est la violence qui traverse l'ensemble du système économique et qui fait de la violence quotidienne un élément constituant de la société chilienne.

Il est fondamental de retenir la distinction posée par la pensé politique contemporaine entre le politique et la politique, car elle permet de définir l'autonomie de l'ordre politique dans sa relation avec d'autres sphères de la vie sociale et d'expliciter la profonde crise politique qu'affronte la démocratie chilienne. ${ }^{4}$ Dans un même temps, tandis que ceux qui prônaient le changement social et le renfort de la démocratie croyaient en une dimension raisonnable du pouvoir, les pouvoirs factuels agissaient en marge des mécanismes institutionnels de la démocratie. Afin de comprendre la tension qui affecte la démocratie chilienne, il est nécessaire de l'envisager sous l'angle de telles oscillations, entre raison politique et pouvoirs factuels, lesquelles configurent la profonde ambiguïté qui marque la société chilienne et produisent des conséquences dramatiques pour beaucoup de sociétés latino-américaines. Pour toutes ces raisons, il nous semble essentiel de préciser les circonstances dans lesquelles la violence devient partie constituante de la dimension politique ; il ne s'agit pas néanmoins d'une violence subjective déterminée par la catégorie de la réflexivité et de la critique, inhérentes à des sujets et des communautés au rôle défini, mais plutôt de formes de violences objectives comme le souligne Zizek. ${ }^{5}$

11 Ces propos autour d'une violence fondatrice du politique doivent être analysés en parallèle avec une phénoménologie de la mémoire qui situe le pouvoir dans une dynamique historique et s'installe dans le souvenir des victimes, des survivants et de ce qui grave la présence de la configuration hétérogène de la violence. En ce sens, l'imposition permanente de groupes dominants à d'autres génère d'une certaine façon une souffrance sociale qui rappelle constamment la destruction de l'ennemi interne. La mémoire de la souffrance sociale se présente comme une dynamique constante des processus de construction et de destruction des modes de coexistence post-dictature. À une autre échelle, il s'agit d'élaborer une phénoménologie capable de retracer le parcours spatio-temporel des pouvoirs factuels, depuis l'arrivée massive des conquérants sur le continent pour ce qui est du passé lointain, jusqu'aux nouveaux pouvoirs de domination plus sophistiqués de la globalité.

Il me semble que l'ouvrage de Fornet-Betancourt Crítica intercultural de la filosofía latinoamericana actual a explicité une série d'observations relatives au caractère fondateur de la politique latino-américaine à partir de la date symbolique de " 1492 ». Nous ne pouvons pas exposer ici tous les tenants et aboutissants du débat mais son issue suggère que la grande proposition politique des processus de libération doit être saisie par la pensée critique afin d'interroger la visibilité effective des pouvoirs factuels pris dans leur contexte et d'associer la violence socioculturelle aux mémoires sociopolitiques. Il s'agit de progresser dans la récupération des mémoires et de faire de la place aux forces de résistances socio-culturelles et politiques tournées vers un nouveau projet proprement américain ${ }^{6}$.

13 Une telle perspective permet de progresser dans la compréhension critique d'un terrain miné par la théorie de l'ambiguïté politique et de se demander comment avancer vers un type de pouvoir libérateur dans le cadre de sociétés asymétriques, en assumant à la fois le lien de domination et les contextes culturels. Ceci implique de sortir du champ, d'une certaine façon pacifique de l'éthique interculturelle, pour saisir la conflictuelle et complexe thématique de la domination prise comme composante essentielle d'une action située, tout en considérant bien entendu le chemin à parcourir 
pour avancer vers une culture de l'émancipation. Ce décryptage du politique est le seul capable de saisir les processus d'hétéro-reconnaissance de sujets et de collectivités jusqu'alors niés dans leur action; quitte à nous répéter, en rejetant le rôle de la violence fondatrice, nous ne cherchons absolument pas à consolider une culture de l'immobilisme et encore moins de la défaite. Il s'agit plutôt de comprendre cette histoire de la violence afin de dé-construire une vision naïve de ces processus de violence vécus et soufferts, de faire apparaître trois figures-clés de la violence historique et de proposer des possibilités dans le cadre d'une politique limitée.

\section{Reconstruire l'histoire du pouvoir et la mémoire des victimes}

Eu égard à ce qui vient d'être signalé, il nous semble qu'une perspective politique de la violence fondatrice de la société chilienne et latino-américaine doit être conçue au delà de ce qui a été qualifié dans le contexte anglo-saxon de «politique de la reconnaissance ». Ceci nous invite à assumer le rôle central tenu par la permanente dialectique de la négation de l'autre exprimée sous une variété de formes violentes et destructives. Ces dernières configurent les structures factuelles du pouvoir qui sont à l'origine des sociétés coloniales et qui ont été maintenues sous la période républicaine. On pourrait dire que l'on retrouve en grande partie dans la structure politique des oligarchies du $\mathrm{xIX}^{\mathrm{e}}$ siècle la façon de construire le pouvoir qui avait été employée pendant la période d'expansion des empires européens à compter du $\mathrm{XVI}^{\mathrm{e}}$ siècle. En effet, la reconstruction du pouvoir postindépendance en Amérique latine ne modifia pas radicalement la composition du pouvoir; de nombreux secteurs de la vie sociale ont ainsi continué à être humiliés et délaissés sans bénéficier de la reconnaissance qui leur était due. Ce fut le sort des indigènes, des paysans et des afro-descendants et il n'est aujourd'hui pas exagéré d'affirmer que depuis plus de cinq siècles, de tels mécanismes discriminatoires secouent et affligent les sociétés en niant aux hommes et aux femmes la valeur et la dignité dont ils devraient jouir en tant qu'être humains et communautés historiques. Il n'est pas non plus exagéré de dénoncer une connexion structurelle entre le pouvoir factuel et les élites, dont les pratiques de violence, dérivées de celles à l'origine de la fondation des empires coloniaux, ont servi à reconstruire les nations conformément à leur idéologie et caractérisé l'ordre dictatorial de l'État défini par l'idéologie de la sécurité nationale.

Cette permanente recomposition du pouvoir factuel dans des sociétés asymétriques exige de repenser, dans une perspective historique, les processus de domination par la violence, non pas comme des évènements sporadiques ou fortuits de la vie politique de nos peuples, mais comme ce que nous qualifions de violence fondatrice; cette dernière détermine le caractère hétérogène et asymétrique des processus de construction politique des institutions latino-américaines qui définissent les structures du nous et des autres, des nationaux et des étrangers, des amis et des ennemis en fonction d'un ensemble sélectif de groupes de familles qui tiennent les "rênes du pouvoir ». Comme nous l'avons déjà signalé, nous nous intéresserons particulièrement à trois "figures ", d'une certaine façon prototypiques- non pas en tant que processus historiques soumis à l'explication d'une discipline scientifique -, mais en tant que "figures » d'une phénoménologie du pouvoir qui servent de support à l'étude de la configuration hétérogène du politique et de la politique latino-américaine. Cette configuration a été 
historiquement définie par la violence contre les dits «barbares ", par l'imposition de groupes dominants (par la domination de groupes sur d'autres?) sur d'autres et par la destruction de l'ennemi interne - antipatriotique -, dans une perspective qui découvre le parcours spatio-temporel de la violence exercée par les pouvoirs de fait. Analysons maintenant certaines caractéristiques de ces trois figures de la violence fondatrice.

\section{1. L'invasion de l'Indo-Amérique et le traumatisme de la conquête} politique latino-américaine débute avec un évènement structurel d'une force extraordinaire: les nouveaux arrivants imposent, sous le poids des armes, les structures européennes du pouvoir à celles alors existantes. C'est le règne de l'ego conquiro. Bien que dans certaines situations les structures amenées par les envahisseurs se sont superposées à celles préexistantes, tous les efforts visaient à légitimer la validité post-factum d'un type de pouvoir militaire et religieux au détriment de l'existant. Dans cette perspective historique, le pouvoir en Amérique latine a presque toujours été défini à partir de son esprit colonial. Dans toutes les sociétés d'Amérique qui ont émergé à partir de ces processus dévastateurs, les différentes colonies espagnoles, portugaises, françaises et anglaises se sont établies en dépossédant les autorités préexistantes de leurs droits légitimes.

conquête, que nous nous garderons de réduire à un ensemble de faits historiques régulés par un "droit des gens", ce dernier intervenant seulement dans une seconde phase de l'« usage de la force ». Dans son sens de fondation et dans une perspective critique, la politique apparaît comme la trame d'institutions édifiées après l'introduction et l'imposition de la machinerie de guerre au sein des territoires indigènes occupés. Dans cette optique, la politique considère les territoires et les corps comme non explorés ; les terres et les gens peuvent être possédés, dominés et détruits. Il s'agit clairement d'un type de pouvoir qui cherche à instaurer un système de domination capable de générer et d'assurer la légitimité de la richesse de l'encomendero, fortune qui enrichira in fine les finances des empires de l'époque et des investisseurs qui réclament le retour de leurs prêts.

Bien entendu ce modèle de pouvoir se déclina de différentes façons au sein du territoire indo-américain; il connut un grand succès à l'intérieur des grands empires théocratiques des vice-royautés naissantes du Mexique et du Pérou, où dès le début, l'habileté de Cortés et de Pizarros leur permit de dominer en superposant leurs institutions aux systèmes précolombiens hautement stratifiés. Dans ces empires préhispaniques, il n'a pas été trop difficile de passer d'un système de contribution annuelle à un système d'impôts nécessaire à la survie du système. En revanche, des efforts beaucoup plus complexes ont dû être déployés pour soumettre les populations autochtones non familières des relations de pouvoirs centralisées ou des systèmes administrés par une caste supérieure.

Dans les diverses configurations coloniales apparaît un lien évident entre les systèmes théocratiques associés à la chrétienté (la croix) qui ont défini et légitimé les limites du pouvoir de l'empire (l'épée). La position de personnes comme Las Casas, qui dénoncent "la destruction des Indes", demeure la plus emblématique de cet imaginaire de la révolte aux débuts de la modernité. Nous savons que la discussion lascasienne traverse 
toute l'Amérique, en particulier le Chili marqué par une tradition héritée de certains évêques et de la figure seigneuriale de $P$. Luis de Valdivia, qui considéraient le système d'encomienda et le « service personnel » comme profondément injustes et qui estimaient que les Indiens avaient droit de bénéficier de leur part. Il va sans dire que l'extraordinaire diminution de la population indienne est imputable à la guerre, la famine et la migration et non à la propagation de maladies. En effet, pendant le premier cycle correspondant à la naissance de la Capitainerie générale, la population indigène diminua de $80 \%$ en cinquante ans ${ }^{7}$.

Une analyse de la politique, telle qu'elle est pensée ces dernières années, en suivant la voie ouverte par la théologie et la philosophie de la libération, permet de reprendre l'optique d'une histoire critique du pouvoir. Les contestations lascasiennes ou valdiviennes sont particulièrement pertinentes pour nos hypothèses dès lors qu'elles permettent de capter cet idéal du pouvoir opposé aux thèses du philosophe Ginés de Sepúlveda reprises par la Capitainerie générale. Ces remises en cause prophétiques ont démontré avec grande force, au-delà de ses conséquences destructrices, le caractère structurant d'une violence fondatrice basée sur le simple pouvoir de l'épée par delà le droit.

\section{2. Civilisation-barbarie et lutte de nationalité}

21 La violence ne sévit pas seulement au moment de l'«invasion initiale » de l'IndoAmérique, comme l'a démontré E. Dussel; elle forge les relations historiques des peuples métisses. La violence armée va perdurer tout au long de la période coloniale, puis revient en scène avec de nouveaux élans au cours des guerres d'indépendance et lors de l'établissement des frontières de l'État-nation. Le conflit belliqueux s'installera dans la durée avec la guerre contre les défenseurs de l'ordre impérial, les guerres aux provinces et aux villages voisins, les guerres contre les indigènes, les noirs et contre tous ceux que menace la constitution du pouvoir étatique naissant. Dans cette perspective, les sociétés indigènes, locales et provinciales, apparaitront comme des obstacles à la délimitation territoriale des États naissants, basée sur la formation d'un pouvoir homogène, ajusté dans la plupart des cas aux préoccupations des groupes dominants de la capitale qui finissent par s'imposer aux intérêts régionaux et locaux.

Néanmoins, tout comme sous la Révolution française, les républiques naissantes ne parviennent pas à se consolider sans souffrir les conséquences de la Terreur. L'indépendance croule sous les jugements et les exécutions sommaires dirigés non pas contre les représentants de l'Ancien Régime, mais contre tous ceux qui n'acceptent pas la logique d'un État édifié à l'image de quelques membres de l'élite au détriment de l'intérêt de tous. Ceux qui ne furent pas exécutés furent déportés; il est du reste notoire que les principaux acteurs de l'indépendance ont fini leurs jours hors du pays qu'ils aidèrent à édifier; un manteau de silence couvre ces histoires en taisant les profonds affronts infligés aux acteurs de l'indépendance.

23 Au nom de la civilisation, une politique d'agression contre le monde indien s'abat sur toute l'Amérique et se concrétise du Nord de l'Amérique à la Patagonie par la mise en place d'une politique concertée entre les États naissants, autour de l'expansion de leurs territoires et le déploiement de processus d'extermination qui rappellent la naissance des colonies. La société mapuche est vaincue par ce que nous connaissons sous le nom de guerre du désert et pacification de l'Araucanie ${ }^{8}$. Le drame de l'invasion des 
territoires indigènes a été documenté au sein de la Comisión de Verdad Histórica y Nuevo Trato. Les trois ethnies patagonnes connurent un sort encore plus tragique en souffrant un véritable holocauste, comme l'atteste l'œuvre magistrale de Martin Gusinde, l'une des meilleures œuvres ethnographiques et de défense des anciens habitants de la Terre de Feu qu'il présente dans toute leur dignité.

\section{3. La défense de l'Occident et la lutte anti-insurgé} dispositifs et pratiques symboliques de la guerre interne définie par la doctrine de la «sécurité nationale - qui est une production des élites militaires des grandes puissance occidentales qui endoctrina de nombreux officiels latino-américains de haut rang dans leur lutte contre l'expansion mondiale du communisme. Cette logique caractérise, en Amérique latine, la troisième et plus horrible violence menée contre les ennemis présumés de la nation, une violence vécue et soufferte il y a tout juste quelques décennies, qui se présente non seulement comme le bornage d'un espace de contrôle territorial contre les ennemis de la patrie mais aussi comme un dispositif sophistiqué destiné au contrôle physique de tous les corps. La pratique systématique et perfectionnée de la torture repose sur un imaginaire de corps rebelles qu'il faut soumettre au moyen de procédés symboliques à mettre en œuvre contre d'éventuels insurgés. Pour les agents, ces insurgés ne sont plus des êtres humains, mais de simples apparences, tout comme autrefois pour les partisans de la Conquête. Cette nouvelle idéologie, qui réduit l'autre à une simple «chose menaçante voire clairement ennemie » conduira à légitimer l'élimination physique de l'autre ainsi que la disparition de toute preuve physique de sa mort.

Dans ces circonstances, il semble opportun de parler du génocide délibéré des dirigeants sociaux et politiques. Il y a encore en Amérique latine beaucoup de personnes disparues et de communautés qui demeurent méconnues par les structures de pouvoir des sociétés majoritaires. Le génocide de plus de 100000 détenus-disparus pendant près de deux décennies est une question qui attend encore d'être saisie par la philosophie politique latino-américaine. Prenons l'exemple d'un pays en particulier pour illustrer nos propos ; les conclusions du rapport final du Documento de la Comisión de Verdad y Reconciliación del Perú, reconnaissent que les victimes indigènes ont été parmi les plus nombreuses:

La CVR a constaté que la tragédie soufferte par les populations du Pérou rural, andin, amazonien, quechua, ashaninka, paysan, pauvre et peu éduqué, ne fut pas ressentie ni assumée comme personnelle par le reste du pays ; ceci confirme, selon la CVR, le voile de racisme et les pratiques humiliantes qui perdurent dans la société péruvienne près de deux siècles après la naissance de la République9.

Pour comprendre un pan significatif de l'histoire latino-américaine, nous devons considérer les règles et les structures du pouvoir qui ont défini le complexe carrefour asymétrique que l'on retrouve dans la logique de la négation permanente présente dans plusieurs pays. Bâtie sur l'inexistence des sujets et des communautés, cette dialectique consacre une relation de subalternité où les sujets niés sont profondément dévalorisés et leurs droits méconnus.

En guise de synthèse, à travers ces trois figures de la violence historique, nous n'élaborons pas une taxonomie historique de la violence mais plutôt un essai philosophique visant à expliciter la structure phénoménologique de la violence afin de 
replacer la mémoire des victimes dans une dimension éthico-politique et d'assurer la restitution de leur dignité d'êtres humains. Il s'agit alors de penser une idéalité qui permet de construire une structure politique différente, qui ne limite pas la relation principale au souvenir de ceux qui furent assassinés et exterminés. En effet, la problématique n'interpelle pas seulement la souffrance d'hommes, de femmes et de communautés entières qui a été causée par la machinerie du pouvoir; elle concerne aussi le legs culturel de ceux qui ont survécu : la souffrance, la douleur et la mort. Il nous semble que la mémoire publique se doit d'honorer symboliquement la violence d'hier, qu'elle doit actualiser et situer en pointant son action et son incidence sur le présent. En l'espèce, nous nous référons à la violence antérieure à l'instauration de tout lien social et historique, à une nouvelle manière de faire de la politique, entre nous, en nous fixant un seuil à dépasser afin d'engager de nouvelles luttes nous permettant de penser un autre type d'humanité. L'hypothèse centrale esquissée dans cette perspective devient intelligible dès lors que la politique et le politique sont de nouveau appréhendés dans le contexte d'une politique latino-américaine de la reconnaissance; une politique de la reconnaissance jamais fermée mais ouverte à de nouveaux défis politiques.

\section{Une politique située de la reconnaissance}

Les idées présentées au sujet des caractéristiques de la violence latino-américaine permettent de reformuler une proposition politique de la reconnaissance, en adoptant une réflexion philosophique différente sur la « critique du pouvoir » dans le cadre des relations modernes de reconnaissance entre subjectivités. Cette analyse riposte, à la manière définie par A. Honneth ${ }^{10}$ mais aussi en recueillant d'autres aspects liés aux droits des communautés dans le monde anglo-saxon comme l'a commenté en particulier Ch. Taylor ${ }^{11}$

$\mathrm{Au}$ sein de cette brève étude, nous ne prétendons pas épuiser toutes les implications de ces théories de la reconnaissance dans leur relation avec le mépris, l'humiliation et la violation de droits, mais il nous semble cependant important de souligner la synergie observée avec ce qui a été élaboré par la pensée latino-américaine actuelle. Il est également possible de rattacher la politique de la reconnaissance à la tradition hégélienne, notamment à la thématique centrale de la « lutte pour l'affirmation du soi » inhérente à la philosophie d'Artur Andres Roig, ou bien à la politique critique de la libération d'Enrique Dussel. On observe aussi une filiation avec la pensée critique interculturelle qui réfléchit à la récupération des mémoires aveuglées, des luttes et des résistances acharnées des communautés de vie ${ }^{12}$.

En ce sens, l'analyse philosophique de la violence systémique du modus operandi de la dictature chilienne s'inscrit dans l'énorme choc contemporain des rationalités en lutte pour le pouvoir et ne peut être réduit aux seuls problèmes vécus par la société chilienne. Ce qu'il nous semble opportun de relever ici est que cette violence fait partie d'un processus systémique visant à astreindre les sociétés engagées dans l'amélioration des processus démocratiques. Dans cette perspective, les dictatures latino-américaines correspondent à une certaine manière de faire de la politique dans laquelle interviennent beaucoup d'éléments transversaux. Par conséquent, il n'est pas possible de réserver la politique de la mémoire aux seules victimes d'un pays, étant donné qu'elle implique des droits violés pendant des siècles dans tous les pays et qu'elle 
répond à une configuration historique particulière des sociétés asymétriques où le pouvoir dépend de la violence structurelle.

31 Sur ce plan du politique et de la mémoire publique, nous souhaitons spécialement souligner la dette de nos hypothèses envers le courant de pensée latino-américain d'aujourd'hui ; comme nous le savons, ces trois dernières décennies ont permis de repenser de manière décisive la tradition philosophique du pouvoir, la mémoire socioculturelle de la violence et le lien existant entre la critique politique et culturelle. Il me semble que dans les trois expressions au sein desquelles la pensée politique se reconnaît d'aujourd'hui, en tant que philosophie latino-américaine, philosophie de la libération et philosophie interculturelle - et ce malgré leurs indéniables convergences, différences et nuances exposées dans l'ouvrage mentionné de Fornet-Betancourt -, celle-ci répond surtout à la redéfinition de la théorie d'une politique critique de la reconnaissance qui vient compléter l'éthique de la libération ainsi que la philosophie politique critique comme l'a qualifiée Dussel.

Une perspective latino-américaine va bien au-delà de ce qui est connu dans le monde anglo-saxon comme "politique de la reconnaissance", car cette pensée assume de manière décisive la dialectique permanente de négation de l'autre qui trouve son expression dans la multidiversité des formes de violation et de destruction qui configurent les structures factuelles du pouvoir et secouent et affligent les sociétés latino-américaines depuis plus de cinq siècles. Ces violences nient la valeur de chaque être humain, de chaque vie humaine potentielle et même des communautés historiques car elles cherchent simplement à garantir la permanence des pouvoirs de fait. Cependant nous ne pouvons pas promouvoir un mode de pensée enraciné dans le défaitisme ou dans l'immobilisme, mais plutôt favoriser un mode de pensée qui transpose la mémoire dans une nouvelle action politique, en consacrant un nouveau sens du futur et de la responsabilité à ce qui a été vécu par le passé.

Dans notre perspective, il s'agit de réélaborer une politique située de la reconnaissance, qui cherche à répondre avec rigueur aux multiples mémoires de la vie sociale et à la violence qui leur est inhérente, où les mémoires de la violence sont insérées dans de nouvelles formes contextuelles de vie, et surtout, qui nous permette de récupérer le sens politique de l'action historique. En ce sens, nous aspirons à l'émergence d'une culture de l'émancipation qui répond à une structure de la domination incarnée. Il s'agit d'une démarche critique qui réagit à l'invisibilité permanente des désaccords et propose un agir solidaire où le souvenir ouvre de nouvelles perspectives permettant d'élaborer un projet politique respectueux des victimes directes et indirectes de la répression. Il s'agit de cette façon d'assumer, d'un point de vue critique, une perspective historico-culturelle, qui, tout en assumant la négation multiple de l'autre (indigène, africain, paysan, femme, pauvre, etc.), permet de répondre à des projets novateurs au service de matrices culturelles émergentes. Formulé ainsi, il s'agit de redéfinir les analyses possibles afin de transformer les échanges asymétriques entre sujets et communautés et projeter de nouvelles formes de reconnaissance des projets politiques.

Une telle politique critique ne se charge pas seulement de rendre explicites certains principes théoriques mais se demande également quelle méthode a permis de dissimuler les relations factuelles établies entre les uns et les autres, d'ignorer la problématisation motivée de l'exclusion des autres, et de méconnaître publiquement le droit à la réparation permettant d'assumer en public la douleur des victimes. En 
assumant le défi majeur posé par l'asymétrie du pouvoir dans nos sociétés, il est possible d'aborder autrement le questionnement des pratiques de la démocratie actuelle en matière d'impartialité et d'inclusion, et d'articuler des principes universalisables afin d'imaginer une autre manière de faire de la politique dans un monde globalisé, au-delà des vieilles et nouvelles formes de violences structurelles. Dans cette perspective, il me semble que la thèse de Fornet Betancourt est importante car elle touche à une thématique centrale de la philosophie politique : la réflexion et la critique autour de la violence invisible, l'absence d'espace-temps consacré à la récupération des mémoires pour les maux soufferts au cours des cinq siècles qui ont défini et articulé les principaux jalons des «nouvelles luttes pour la reconnaissance ».

Ce défi de repenser la politique de la reconnaissance ne nous force pas seulement à sortir de cette violence structurelle afin de ne pas rester prisonniers de faits produits il y a quarante ans, mais nous pousse à nous détacher du passé proche et lointain afin de réaliser que le problème tient précisément à la question de savoir comment avancer dans la résolution de grand nombre de ces violences codifiées dans le système économique et politique contemporain. Un souvenir philosophique du coup d'État militaire, quarante ans après, nous permettrait de capter la complexité de notre présent politique à partir de l'expérience des victimes, et de saisir un nouveau mode de faire de la politique dans des contextes culturels où la domination extra et intra culturelle leur est consubstantielle. Cela permettrait par ailleurs à la philosophie politique de se détacher d'autres exigences pour ainsi pouvoir critiquer la permanence des pouvoirs hégémoniques et développer des mécanismes capables de retourner ces dynamiques d'invisibilisation tenaces. Dans cette optique, il convient de reconnaître que la philosophie politique est un art à la fois théorique mais aussi éminemment pratique, inséré dans des contextes sociaux, politiques et culturels où sont élaborées les trames du discours et du pouvoir. Cette nouvelle pensée politique assistée par une herméneutique critique et, surtout, par une philosophie politique interculturelle dépasse cette culture du silence et ce qui est défini comme violence et domination chronique. Il n'est plus possible d'esquiver l'enjeu de la construction d'un espace commun au-delà d'un vide interculturel, lié à la logique de la négation des sociétés, des cultures et des corps. En cela réside selon nous le principal défi de la politique latinoaméricaine. En effet, la philosophie politique latino-américaine n'est pas encore parvenue à assurer - dans cette riche complexité qui caractérise la pensée critique latino-américaine -, un apport substantiel, à savoir que le démontage des systèmes de domination et des fonctions idéologiques associées au pouvoir s'obtient toujours par la conquête graduelle de la réflexivité et de la critique liées aux "subjectivités émergentes » mais aussi aux pratiques politiques des organisations socioculturelles.

\section{Pour ne pas conclure...}

Une philosophie politique à l'écoute de la pensée critique doit rendre compte du double mouvement intégrateur et désintégrateur par lequel opèrent les mécanismes actuels de rencontre et de non-rencontre. Cela exige d'assumer que le terrain de la récupération de la mémoire est un terrain en permanente dispute, où se jouent des conflits inhérents à l'imaginaire des communautés et à différents projets de vie sociale. Les configurations politiques d'une société concrète montrent que la politique tend toujours à être réduite à la gestion des pouvoirs, des privilèges et de reconnaissances non horizontales; ce qui 
nous introduit dans le champ de l'asymétrie historique des communautés et des sociétés. L'histoire du pouvoir renvoie presque toujours à la lutte de groupes humains et de sociétés puissantes contre des groupes et des sociétés plus faibles, comme l'illustre très clairement le traitement injuste et violent infligé par les conquérants et nos élites oligarchiques aux régions et aux communautés indigènes, afro-américaines et pauvres d'Amérique. Mais la politique, avec sa charge dramatique, ne représente pas un tout, car le conflit apparaît comme le terrain d'une rationalité idéale où se joue la gestion de la mémoire du conflit et le respect des victimes en tant que composantes substantielles de la vie culturelle et politique.

À ce niveau d'ambivalence entre la politique et le politique, le projet de reconnaissance se place au milieu d'une tension tangible entre inclusion-exclusion, mémoire et oubli, ce qui nous amène à démontrer que, dans cet exercice, se joue la possibilité de saisir le paradoxe entre les traditions contextuelles auxquelles elle donne origine. On pourrait compléter ces analyses en concluant que la « violence fondatrice » ne dévalorise en rien le labeur politique, entendue comme une action sociale au sens historique et non comme un simple instrument des pouvoirs de fait. In fine, au-delà de la violence et de la guerre, c'est justement le politique qui permet d'aborder l'espace des savoirs contextuels et locaux en vue d'une articulation chaque fois plus universelle et suffisamment critique, où la mémoire des victimes pourrait être pleinement honorée. En conséquence, ce qui permet d'exiger la reconnaissance éthico-politique de l'holocauste juif doit permettre de la réclamer pour l'holocauste des indigènes et des Africains en Amérique.

Dans l'esquisse de ces trois figures de la violence, nous ne cherchons pas à replacer au cœur des discussions la taxonomie historiciste de la violence mais plutôt à suggérer une phénoménologie du pouvoir qui rend à la mémoire des victimes son importance éthicopolitique pour l'ensemble de l'histoire de l'Amérique. Il s'agit ici de penser la politique d'une grande patrie où il est possible de faire mûrir le lien fondamental existant entre les diverses luttes émancipatrices, révolutionnaires et de résistance, au nom desquelles ont été projetés des mondes sociaux nouveaux mais ont aussi été assassinés et exterminés par la machinerie des pouvoirs factuels des hommes, des femmes et des communautés entières. Ces derniers méritent d'être reconnus non seulement en tant que composantes d'un héritage moral et politique, mais aussi en tant que legs des traditions de luttes et de résistances qui permettent de renouveler l'énergie de l'engagement politique et social. La nouvelle culture politique se trouve symboliquement enracinée dans une violence d'hier tout en se projetant dans une redéfinition du politique situé dans le présent et nous permettant de pratiquer une politique entre nous, dans le scénario de la société émergente à venir. La perspective politique de la reconnaissance que nous déployons ici ne dérive certainement pas d'une lecture des textes actuels de la philosophie politique mais est issue de la dynamique des savoirs localisés, ouverts à la fois sur la problématique de la mémoire latino-américaine et sur les processus sociopolitiques d'intégration. Reconnaissons alors notre dette envers une expérience étroitement liée à l'histoire politique récente du pays dans lequel nous vivons et reconnaissons ainsi les dettes historiques contractées envers tous ceux qui ont souffert; en particulier, celles qui interpellent la conflictuelle problématique relative aux nécessaires limites du pouvoir politique et les efforts que la démocratie doit déployer afin de reconstruire une mémoire publique qui aille au delà du cadre du bicentenaire. Ce défi théorico-pratique, à notre sens, constitue l'unique 
point de départ pour cette possible inclusion de l'autre que suggère la politique de la reconnaissance ; aller au delà de la logique et de la machine de guerre interne.

\section{BIBLIOGRAPHIE}

Balibar Étienne, La crainte des masses, Paris, Éditions Galilée, 1997.

Bauman Zigmun, In search of politics, London, Polity Press \& Blackwell Publishers Ltd, 1999.

Canio Margarita et Pozo Gabriel (ed.), Historia y Conocimiento oral mapuche, Santiago, éditions LOM, 2013.

Comisión de Verdad y Reconciliación del Perú, Conclusiones n 9, http://cverdad.org.pe/ifinal/

De Ramón Armando, Historia de Chile, Santiago, Catalonia, 2003.

Ferry Jean-Marc, Les puissances de l'expérience, Paris, Éditions du Cerf, 1991, 2 tomes.

Fornet-Betancourt Raúl, Transformación intercultural de la filosofía, Bilbao, Desclée de Brouwer, 2003.

Fornet-Betancourt Raúl, Crítica intercultural de la filosofía latinoamericana actual, Madrid, Ed. Trotta, 2004.

Honneth Axel, Kampf um Anerkennung-Zur moralischen Grammatik sozialer Konflikte, Frankfurt am Main, Suhrkamp, 1992. (Trad. Castellana 1997).

Marchant Oliver, Post-Foundational Political Thought, Edinburg University Press, 2007.

Roig Arturo Andrés, Teoría y Crítica del pensamiento latinoamericano, México, FCE, 1981.

Salas Ricardo, Lo Sagrado y lo Humano, Santiago, San Pablo, 1996.

Salas Ricardo, "Filosofía intercultural, Políticas del Reconocimiento y Violencia inter-étnica en Tierras Mapuches (Chile)", in Alteridade Peregrina, G. Meinhardt (ed.), São Leopoldo, Nova Harmonia-OIKOS Editora, 2008, p. 96-106.

Salas Ricardo, "Para pensar tópicos e temporalidades do encontro-desencontro na filosofia intercultural”, in Pensamento critico IV : Mundo da vida, Interculturalidade e Educação, J. Pizzi, Pelotas, Ediciones UFP, 2012, p. 119-138.

Salas Ricardo, Salvat Bologna Pablo, "Del autoritarismo y la interminable transición : notas sobre la discusión de la democracia en Chile", Revista de Ciencia Política de la UN de Colombia, n 7, 2009, p. 89-112.

Taylor Charles, Multiculturalism and the Politics of Recognition, Princeton, 1992. (Trad. Cast. 1997).

Zizek Slavoj, Violence, London, Profile Books LTD, 2008. 


\section{NOTES}

1. Voir nos travaux: Salas Ricardo, "Filosofía intercultural, Políticas del Reconocimiento y Violencia inter-étnica en Tierras Mapuches (Chile)", in Alteridade Peregrina, G. Meinhardt (éd.), Sao Leopoldo, Nova Harmonia-OIKOS Editora, 2008, p.96-106; et "Para pensar tópicos e temporalidades do encontro-desencontro na filosofia intercultural", in Pensamento critico IV: Mundo da vida, Interculturalidade e Educação, J. Pizzi, Pelotas, Editions UFP, 2012, p. 119-38.

2. À ce sujet, voir notre ouvrage Lo Sagrado y lo Humano, Santiago, San Pablo, 1996, p. 62.

3. Marchant O., Marchant Oliver, Post-Foundational Political Thought, Edinburg University Press, 2007.

4. Voir les textes de É. Balibar, La crainte des masses, Paris, Éditions Galilée, 1997 ; Z. Bauman, In search of politics, London, Polity Press \& Blackwell Publishers Ltd, 1999 ; ainsi que de J. M. Ferry, Les puissances de l'expérience, Paris, Cerf, 1991, 2 tomes; et notre "Del autoritarismo y la interminable transición: notas sobre la discusión de la democracia en Chile", Revista de Ciencia Política de la UN de Colombia, $n^{\circ}$ 7, 2009, p. 89-112, co-écrit avec Pablo Salvat Bologna.

5. Nous nous référons en particulier aux thèses élaborées par S. Zizek, Violence, London, Profile Books LTD, 2008.

6. Voir la proposition de R. Fornet-Betancourt, Transformación intercultural de la filosofía, Bilbao, Desclée de Brouwer, 2003.

7. De Ramón Armando, Historia de Chile, Santiago, Catalonia, 2003, p. 26.

8. Nous recommandons la lecture des témoignages des survivants de la guerre du désert et de l'occupation de l'Araucanie, dans l'ouvrage édité par M. Canio et G. Pozo, Historia y Conocimiento oral mapuche, Santiago, éditions LOM, 2013.

9. Document final de la Comisión de Verdad y Reconciliación del Perú, Conclusiones n 9. L'intégralité du rapport est disponible sur http://cverdad.org.pe/ifinal/

10. A. Honneth, Kampf um Anerkennung-Zur moralischen Grammatik sozialer Konflikte, Frankfurt am Main, Suhrkamp, 1992. (Trad. Castillan 1997).

11. Ch. Taylor, Multiculturalism and the Politics of Recognition, Princeton, 1992. (Trad. Cast. 1997).

12. Fornet-Betancourt, Crítica intercultural de la filosofía latinoamericana actual, Madrid, Trotta, 2004.

\section{RÉSUMÉS}

Cet article développe une thèse philosophique autour de la violence fondatrice des processus politiques latino-américains. Dans cette perspective, nous ne nous focalisons pas exclusivement sur la dictature militaire mais proposons plutôt une hypothèse générale sur la "violence fondatrice » des sociétés d'Amérique latine, en prenant pour référence les nombreux usages de la force survenus au cours des dix-sept difficiles années vécues par la société chilienne - et pour une plus longue durée dans le cas des autres pays latino-américains. Ce cadre permet de comprendre, à partir d'une phénoménologie historique, les violences et les cruautés générées par la dictature chilienne. Notre propos principal suggère que le Coup militaire de 1973 n'est ni un fait violent imputable au hasard, ni un phénomène nouveau, en ce qu'il s'insère dans la " logique de la négation permanente » inscrite dans la société chilienne et qui a marqué le rapport spatiotemporel de ses relations sociales; en ce sens, elle affecte non seulement la mémoire du court et long terme, mais aussi la problématique de la réparation des victimes, deux thèmes qui 
intéressent la politique de la reconnaissance. Pour affronter ces questions, il apparaît nécessaire de proposer une perspective philosophique de la violence fondatrice de la politique en opposition au politique.

This article develops a philosophical thesis about violence foundational American political process, and therefore is not a direct pose about the military dictatorship, in other words, we propose a general hypothesis about the "founding violence" formed societies in Latin America, and determines much force facts that occurred in those hard 17 years experienced by the Chilean society and many more in other Latin American versions. This background can understand from a historical phenomenology resulting violence and cruelty of the Chilean dictatorship. The main idea behind is that the 1973 military coup is not a random violent act or a new phenomenon, but it is part of an ongoing "logic of negation" present in Chilean society, which has marked the space-time of sociability ours, and therefore refers both to a question connected with memory both long and short duration as the reparation of victims that is part of a politics of recognition. Thus, it is necessary to propose a philosophical view of the founding of political violence, as opposed to the political.

\section{INDEX}

Mots-clés : violence, politique, reconnaissance, victimes

\section{AUTEUR}

\section{RICARDO SALAS ASTRAIN}

Ricardo Salas Astrain est docteur en philosophie de l'Université de Louvain, Belgique, où il a défendu la thèse « Lenguaje, Hermenéutica y Símbolo Religioso » (1989). Il est actuellement professeur à la Faculté des sciences sociales UC Temuco et chercheur principal à CONICYT. On lui doit notamment : Lo Sagrado y lo Humano (Santiago 1996) et Ética Intercultural (2003) ainsi que la traduction de La Articulación del Sentido de Jean Ladrière (2001). Il a coordonné Pensamiento crítico latinoamericano (2005) et édité Sociedad y Mundo de la vida (2007) et Éticas convergentes en la encrucijada de la postmodernidad (2010).rsalas@uct.cl 